\title{
Oblique plane microscopy-based 3D plate- reader investigation of control of cell shape by Rho-regulatory proteins in distinct microenvironments
}

Nathan Curry $^{1}$, Lucas Dent ${ }^{2}$, Hugh Sparks ${ }^{1}$, Vicky Bousgouni ${ }^{2}$, Vincent Maioli $^{1}$, Sunil Kumar ${ }^{1}$, Chris Bakal ${ }^{2}$, Chris Dunsby ${ }^{1}$ $1_{\text {Imperial College London, United Kingdom. }}{ }^{2}$ The Institute of Cancer Research, United Kingdom

\section{Abstract Text}

Living cells exert precise control over their geometry, responding both to their environment and intracellular signalling. We investigate cellular 3D shape control by Rho-regulators in a homogenous chemical environment but with distinct physical environments.

Using an oblique plane microscope (OPM) [1] we imaged fixed cells plated in collagen in a 96-well plate. This assay allowed the identification of characteristic shape changes that occur as the position of the cell nucleus transitions from a stiff (cell nucleus is close to the coverslip) to soft (cell nucleus is far from the coverslip) physical environments.

Using this method $\sim 200$ cells per well were imaged across 3 multiwell plates. To analyse the data, automated 3D segmentation methods were investigated. We verified that our shape measurements were robust across two different segmentation methods (thresholding using Otsu's method and an active contour segmentation). We also tested our measurements when taking into account the anisotropic and spatially varying OPM PSF, showing the measured shape changes were larger than these effects.

Using cluster analysis, we identified five key cell and nucleus shape parameters. In control conditions four of these conditions (cellnucleus orientations, cell eccentricity, cell protrusiveness and cell 
surface area) vary between cells that have a nucleus positioned close to the coverslip and cells with their nucleus in the collagen. We further identified four RhoGTPase knockdowns that reduce cell protrusiveness near the coverslip relative to control. Notably, we find that whilst one of these knockdowns also controls cell protrusiveness in soft conditions (far from the coverslip), the other three appear to be context specific.

The automated OPM plate reader allows 3D imaging of large numbers of cells and conditions. Recent developments on the system have allowed us to perform time-lapse high-content imaging of cells embedded in gel. We build upon our fixed cell study through time-lapse imaging of 30 independent conditions imaged every 5 minutes for 12 hours, in the same experiment. 